\title{
FIRST-YEAR VERSUS FOURTH-YEAR AND ONLINE VERSUS PAPER-AND-PENCIL RESPONSES TO A GRAdUATE ATTRIBUTE SELF-ASSESSMENT TOOL AND EXIT SURVEY
}

\author{
Andrew J. B. Milne, Roydon Fraser, and Natalie Chow \\ Mechanical and Mechatronics Engineering, University of Waterloo, Waterloo, Ontario, Canada \\ ajbmilne@uwaterloo.ca, rafraser@uwaterloo.ca, natalie.chow@uwaterloo.ca
}

\begin{abstract}
A$ tool has been created that allows students to self-assess how well their program helped them achieve CEAB graduate attributes. These indirect self-assessments are used at the University of Waterloo in the Mechanical and Mechatronics Engineering (MME) Department along with more direct measurements to inform analysis of programs. The tool uses an online survey platform to allow for randomized presentation of a subset of prompts related to the graduate attributes to each student in order to reduce the number of questions posed to any one student while ensuring coverage of all attributes.

This tool has been deployed to first-year and fourth-year cohorts, and the differences in students' self-assessments are discussed, in particular the increase in self-awareness that is expected to occur between first and fourth-year. The tool has also been deployed as part of an online exit survey administered to fourth-year students only, and similarities and differences in survey responses for online versus paper-and-pencil are discussed especially as they relate to free-form responses.
\end{abstract}

Keywords: Self-Assessment, (Exit) Survey, Paper-andPencil versus In-Person-Online, Graduate Attributes

\section{INTRODUCTION}

At their best, measurements of the CEAB Graduate Attributes (GA) lead to efforts to improve an engineering program. In this way, they are akin to other program improvement activities such as exit (and entrance) surveys. In this paper, we present results from a combination of a student self-assessment tool for GAs and an online exit survey. Given that the tool is for self-assessment, the tool and exit survey are also similar in that they are indirect measures. That is, they measure student perceptions of their abilities/the program, rather than being direct measures of students' knowledge and skills, or direct observations of their behavioural tendencies. Both indirect and direct measurements are important in GA assessment to gain a more complete picture of a program [2].
It is also desirable - at least within the engineering departments of the University of Waterloo - to measure GA both near graduation (in analogy to summative assessments) and earlier in each program (in analogy to formative assessments). Therefore, we have administered the same self-assessment tool to 1A (first term) Mechanical (ME) and Mechatronics (MTE) classes, as well as to their colleagues in the 4B (final term) of ME and MTE.

The tool developed makes use of an online survey platform (Qualtrics ${ }^{\mathrm{TM}}$ ) for administration, and to allow for a randomized subset of prompts to be presented to each student. It was administered in a standalone fashion to $1 \mathrm{~A}$ students in the fall 2015 (F'15) term. It was presented in combination with an exit survey to $4 \mathrm{~B}$ students in the winter 2016 (W'16) term as an online-in-person survey (i.e. administered electronically but in a set place and time). This format was in contrast to previous years where the exit survey was presented with little to no inclusion of GA assessment, and as a paper-and-pencil (PAP) survey.

As such, there were concerns raised within the department as to whether the inclusion of the GA selfassessment questions, and the switch from paper-andpencil administration to electronic, would unduly impact the amount and quality of feedback gained from the exit survey. This paper, therefore, explores two areas: first, it analyzes the quantity and quality of data obtained from previous year's PAP exit surveys and the most recent electronic online-in-person offering of the exit survey; and second, it makes a preliminary/pilot analysis of the programs' teaching of GAs as measured by $1 \mathrm{~A}$ and $4 \mathrm{~B}$ student self-assessments to determine if the tool as developed can provide meaningful data going forward.

This paper proceeds first with a review of the literature on PAP versus online surveys, then presents the methodology by which the online-in-person exit survey and GA self-assessment tool was constructed and administered. These results are preliminary in extent and analysis, but it is hoped that the methodology and findings will be applicable to others who wish to conduct investigations of their programs this way. 
A number of studies have been conducted with the purpose of evaluating survey methodologies and examining the differences between PAP and online surveys. These studies have occurred in various fields and have explored key survey research topics such as response rates $[6,7,9]$, response quality $[1,3,5]$, and survey completeness $[3,4]$. However, few studies exist that have investigated the effects of survey delivery in an educational setting [5]. Moreover, while several studies have attempted to identify the advantages and drawbacks of both PAP and online surveys, there remains a lack of consensus with regard to an optimal method of survey administration.

The overall trend in findings related to PAP and online surveys have repeatedly suggested a general equivalence between the two data collection methods [1]. With regard to auxiliary factors associated with measuring equivalence, some studies have proposed that online surveys tend to contain fewer missing responses [4]. Specifically, a study which examined the influence of experimenter presence on $\mathrm{PAP}$ and online survey administration found that questions located at the end of the survey were more frequently skipped by respondents in the PAP condition versus the online version [9]. Another recent study that tested the impact of survey delivery conditions on college students' conceptions of academic and non-academic writing found that the lab online group (with researcher presence, what we call online-in-person here) provided the longest responses compared to their counterparts in the PAP and home online groups. Conversely, studies have proposed the negative impact of researcher presence as a determinant for increasing social desirability effects [5,9] (though this effect would also be present for a PAP survey with such an authority figure present). The quality of responses to openended questions have also been deemed by many researchers as comparable with minor variance $[4,5]$.

This paper outlines preliminary findings from online surveys that were administered via both online-in-person and solely online formats. While studies have shown that PAP surveys tend to elicit significantly higher response rates $[4,6]$, some researchers have advocated for the effectiveness of online surveys with the purpose of collecting feedback from college students pertaining to their experiences and attitudes [5]. Further, it has generally been seen that online-in-person surveys have a response rate closer to that of a PAP in person survey, so it was hoped here that the online-in-person self-assessment tool and online survey would yield useful results.

\section{Methodology}

\subsection{Platform}

As mentioned, the self-assessment tool and exit survey were developed and administered with a commercial licence of the Qualtrics ${ }^{\mathrm{TM}}$ software in F'15/W'16. The software was chosen after an investigation of free and paid survey tool options, with a focus on ease-of-creation, easeof-administration, robust commercial support, ability to collect anonymous or non-anonymous data, and ability to randomize questions and blocks of questions (including with non-equal weighting). Non-anonymous capabilities were desired for future longitudinal assessment of students as they progress through the program, and matched-pair assessment of students' self-assessment and direct assessments. Randomization was necessary to allow for a random subset of the multiple GA/indicators to be selfassessed by each student so as to maintain coverage of all GA without unduly burdening students. Accessibility was also a key consideration in this decision-making process, as students are more likely to value surveys that are easily accessible [6]. In this regard, the cross-platform feature in Qualtrics $^{\mathrm{TM}}$ (i.e. optimized for both mobile devices and laptops) accommodated respondents by capitalizing on the increased flexibility in learning environments [6]. The ultimate decision to pursue a Qualtrics ${ }^{\mathrm{TM}}$ license was based on its meeting constraints at the lowest price per seat.

\subsection{Graduate Attribute Self-Assessment Tool (and Exit Survey) Construction}

The GA self-assessment tool was constructed based on rubrics that have been generated for each $\mathrm{GA} /$ indicator by committees internal to MME. It should be noted that the rubrics are in a preliminary stage and need validation. In modifying the rubrics for self-assessment, each indicator was reworded to be a prompt to the student. Likewise, the related descriptors of beginning, progressing, meeting, and exceeding levels were reworded to be active voice first person statements wherever possible. For example, for the lifelong learning GA, the indicator "Identify sources for new knowledge" and level descriptor "Identifies different knowledge sources available" (progressing level) was reworded to "In searching for new knowledge, I identify different knowledge sources available". The option, "I have not seen this concept / I don't know" was added as a response to each prompt as a lowest level choice.

After each indicator prompt, a further question was posed to students to ask them where they thought they could develop this attribute (1 A students) or to ask them how much they had improved on this attribute (4B students). Both groups of students received two questions at the end of the self-assessment asking them to rate the validity and clarity of the GA prompts on a 5-point Likerttype scale, with "I don't know" as an additional option.

As each indicator was turned into a survey question prompt, there were 34 and 43 prompts in the ME and MTE self-assessment tools, respectively. This was deemed to be too many questions for one student to complete in a single sitting. As such, we designed the survey flow using Qualtrics ${ }^{\mathrm{TM}}$ tools to randomly assign 5-7 questions to each student as they took the survey. The software was designed to balance incoming survey starts in real time to attempt to 
maintain even coverage of each indicator (accounting for the possibility that students may choose to not complete the survey leading to a loss of the self-assessment point). Further, the survey flow was designed such that a single student was given a range of indicators (rather than all the indicators for one GA). This was done with the aim of randomized sampling of each GA/indicator, giving a snap shot of the program as a whole and of students' perceptions of clarity and validity of the indicators.

The exit survey was constructed to be as similar to the PAP survey of previous years as possible, including identical or similar wording of questions, similar ordering of questions (with the change that all demographic questions were consolidated at the end of the survey), and with a similar setup of groups of questions visible on the screen at one time (in comparison to groups of questions visible on the page). This was done to try and maintain as much comparability between previous PAP data and current online-in-person data as possible.

\subsection{Entrance (1A) Administration}

The self-assessment tool was administered to the $1 \mathrm{~A}$ ME and MTE classes (two cohorts each of approximately 100 students for a total of 400 students) in F' 15.

For the ME class, the tool was administered purely online (emailed to the entire class with no specific time set aside to complete the survey) with explanations of the purpose of the exercise and multiple requests to complete the survey given through email and during class time by their Introduction to Engineering course instructor and the authours. The tool was administered near the end of the term. A focus group was held in the W'16 term for those students who had not yet left for work term.

For the MTE class, the tool was administered online-inperson. All students received the survey by email regardless of their attendance on the day of the event, but a lunch hour was booked free for the students in the middle of the term (just after midterms) to come to a separate classroom to complete the survey on their own devices. Students were encouraged to attend by their Introduction to Engineering course professors and by the authours. The purpose of the exercise was explained in class and again at the start of the event. Students were also encouraged to attend through the offer of a free pizza and pop lunch and focus group discussion following the survey. Due to the limited scope of this paper, focus group responses are not included in this paper.

All groups were informed that their participation was voluntary and appreciated, that impact on grades would be non-existent (due to separation of who administered the tool from those grading students), and that results would be kept anonymous and used to improve their programs. Further, the connection of the tool to CEAB requirements was outlined, but with an emphasis that the CEAB does not want to see (falsely) that their programs are perfect, but rather that their program is working towards a process of continual improvement. Finally, the 1A students were informed that the questions asked related to the expected performance of a graduating student. This explanation was given intentionally to try and combat the bias/despair that might result if the levels seemed unreasonably high.

\subsection{Exit Survey (4B) Administration}

The self-assessment tool was administered to the 4B ME and MTE classes (two cohorts and one cohort respectively of approximately 100 students each for a total of 300 students in two programs) in the last week of the W'16 term (after the fourth-year design project symposium was complete). An online-in-person event, plus a pizzalunch and post-survey focus group, was held separately for both groups one the same day, with a further enticement of a limited number of $\$ 50$ gift cards to be randomly awarded to students who completed the survey. All students received a link to the survey by email regardless of their attendance at the event. The event was advertised at the fourth-year design project symposium, and by MME faculty and staff (a more difficult endeavor given the large number of elective courses in the 4B term). A similar preamble was given to students, without the statement about level of expectations being at fourth-year level.

\section{Results and Discussion}

\subsection{Response Rates and Contents}

The combination of tool administration strategies for the 1A ME and 1A MTE programs allows us to examine the effects of purely online (ME) versus online-in-person (MTE) administrations. The combination of the W'16 4B exit survey and previous years exit surveys allows us to examine the effects of online-in-person versus PAP (in person) survey administration. Table 1 shows high-level data for each case.

Table 1 suggests that a purely online administration (1A $\mathrm{ME}$ ) gives the poorest response rates, highest rate of survey termination, and highest length of time spent on the survey, despite the 1A GA self-assessment tool being shorter than the 4B exit survey $(20 \%, 34 \%$, and 36 minutes, respectively), though it should be noted that this cohort also received no incentive for participation. It is also seen that 1A 2015 MTE students and 4B 2016 ME and MTE students (in 2016) exhibited similar response rates for the online-in-person plus pizza lunch focus group administration (34\%-40\%), despite the added incentive of the gift card draw for 4B students. The 4B 2015 data is anomalous in terms of rates since it was administered in a last-minute fashion due to factors outside the control of the Department. Pre-2015 4B data shows much higher response rates $(65-87 \%)$. This is likely due to the administration of the survey during a mandatory safety 
lecture near the start of term (though the survey was optional and incentivized by a gift card draw).

Overall, although factors were not controlled between all administrations, it seems that the largest response rates are not seen via the incentive of pizza, but instead through compulsory attendance (unsurprisingly). Sadly, given the differences in other factors, no firm conclusion can be drawn on the effect of online administration versus PAP, however, online administration does not seem to unduly impact completion rate for surveys which have been started. Indeed, an approximate count of attendance numbers at the online-in-person event suggests that the vast majority of students attending the event completed the survey, while the low percentage of late starts suggests that the in-person event was instrumental in gaining responses.

Table 1: Completion statistics of various cohorts.

\begin{tabular}{|c|c|c|c|c|c|c|c|c|c|c|c|}
\hline & $\frac{\grave{\partial}}{0}$ & $\begin{array}{l}\frac{10}{5} \\
\frac{1}{2}\end{array}$ & 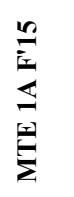 & 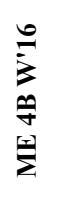 & 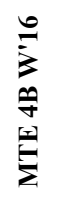 & 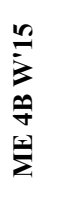 & 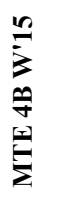 & 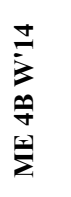 & 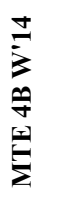 & \begin{tabular}{l}
$m$ \\
$\sum$ \\
\multirow{2}{*}{} \\
$\sum$
\end{tabular} & 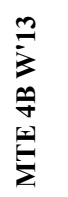 \\
\hline \multicolumn{2}{|l|}{ Cohort population } & 219 & 199 & 173 & 125 & 158 & 113 & 160 & 114 & 122 & 96 \\
\hline \multicolumn{2}{|l|}{ Surveys started } & $\begin{array}{r}62 \\
29 \%\end{array}$ & $\begin{array}{r}94 \\
47 \%\end{array}$ & $\begin{array}{r}69 \\
40 \%\end{array}$ & $\begin{array}{r}59 \\
47 \%\end{array}$ & - & $\begin{array}{l}- \\
-\end{array}$ & - & $\begin{array}{l}- \\
-\end{array}$ & - & $\begin{array}{l}- \\
-\end{array}$ \\
\hline \multicolumn{2}{|c|}{ Surveys completed if started } & $\begin{array}{r}41 \\
66 \%\end{array}$ & $\begin{array}{r}80 \\
85 \%\end{array}$ & $\begin{array}{r}58 \\
84 \%\end{array}$ & $\begin{array}{r}50 \\
85 \%\end{array}$ & $\begin{array}{r}36 \\
-\end{array}$ & $\begin{array}{r}30 \\
-\end{array}$ & $\begin{array}{r}104 \\
-\end{array}$ & $\begin{array}{r}87 \\
-\end{array}$ & $\begin{array}{r}106 \\
-\end{array}$ & $\begin{array}{r}75 \\
-\end{array}$ \\
\hline \multicolumn{2}{|l|}{ Surveys incomplete } & $\begin{array}{r}21 \\
34 \%\end{array}$ & $\begin{array}{r}14 \\
15 \%\end{array}$ & $\begin{array}{r}11 \\
16 \%\end{array}$ & $\begin{array}{r}9 \\
15 \%\end{array}$ & - & $\begin{array}{l}- \\
-\end{array}$ & $\begin{array}{l}- \\
-\end{array}$ & $\begin{array}{l}- \\
-\end{array}$ & $\begin{array}{l}- \\
-\end{array}$ & - \\
\hline \multicolumn{2}{|c|}{ Total response rate (completed surveys) } & $19 \%$ & $40 \%$ & $34 \%$ & $40 \%$ & $23 \%$ & $27 \%$ & $65 \%$ & $76 \%$ & $87 \%$ & $78 \%$ \\
\hline \multicolumn{2}{|l|}{ Duration mean (h:mm) } & $1: 26$ & $1: 31$ & $0: 47$ & $0: 35$ & - & - & - & - & - & - \\
\hline \multicolumn{2}{|l|}{ Trimmed mean (h:mm) } & $0: 36$ & 0:08 & $0: 17$ & $0: 22$ & - & - & - & - & - & - \\
\hline \multicolumn{2}{|c|}{$\begin{array}{l}\text { Late starts (starts after scheduled } \\
\text { admistration) }\end{array}$} & $\begin{array}{r}5 \\
2 \% \\
\end{array}$ & $\begin{array}{r}6 \\
3 \% \\
\end{array}$ & $\begin{array}{r}16 \\
9 \% \\
\end{array}$ & $\begin{array}{r}10 \\
8 \% \\
\end{array}$ & $\begin{array}{l}- \\
-\end{array}$ & $\begin{array}{l}- \\
- \\
\end{array}$ & $\begin{array}{l}- \\
-\end{array}$ & - & $\begin{array}{l}- \\
-\end{array}$ & - \\
\hline$*$ & \multicolumn{11}{|c|}{ Cells marked with '-' were unrecorded/incalculable from previous years } \\
\hline Program/Cohort & \multicolumn{11}{|l|}{ Notes } \\
\hline ME $1 \mathrm{~A} \mathrm{~F}^{\prime} 15$ & \multicolumn{11}{|c|}{ Administered online (introduced in class) Nov. $24+25,2015$} \\
\hline MTE $1 \mathrm{~A} \mathrm{~F} \mathrm{~F}^{\prime} 15$ & \multicolumn{11}{|c|}{ Administered online-in-person with pizza lunch Nov. $11+12,2015$} \\
\hline ME 4B W'16 & \multicolumn{11}{|c|}{ Administered online-in-person with pizza lunch and gift card draw Mar. 31, 2016} \\
\hline MTE 4B W'16 & \multicolumn{11}{|c|}{ Administered online-in-person with pizza lunch and gift card draw Mar. 31, 2016} \\
\hline 4B W'15 & \multicolumn{11}{|c|}{ Administered PAP-in-person with gift card draw Second last week of term } \\
\hline $4 \mathrm{~B}$ pre $\mathrm{W}^{\prime} 15$ & \multicolumn{11}{|c|}{ PAP-paired with mandatory safety lecture with gift card draw Second week of term } \\
\hline
\end{tabular}

\subsection{Qualitative (thematic analysis) and quantitative review of students' comments}

To study the quality of responses, we considered the responses of students to the final open-ended question on the survey, prompting students to "Please provide any other comments about your program or your experience at UWaterloo." Specifically, we conducted a qualitative review of all free-form comments from 2014-2016 (the 2013 exit survey did not include the final general comments question). We are in the process of conducting a formal thematic analysis of the texts. Initial results suggest that similar themes have been expressed by 2016 4B students as in previous years. As a first visualization of themes, we present here a word cloud illustrating the top 40 most common words used by students (Fig. 1).

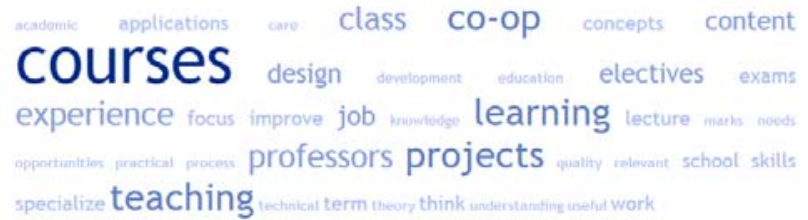

Fig. 1. Top 40 words in 4B exit survey with non-specific words such as 'Waterloo' and 'Engineering' removed, minimum frequency (5). ${ }^{*}$ Created on www.tagcrowd.com

As demonstrated by the word frequency data, a number of common themes emerged from students' comments throughout the 2014-2016 cohort years. The largest text represented, "courses," reflects students' repeated comments on factors related to the program structure and curriculum. Some examples of elements within the 
umbrella term of "courses" include electives, course content, program flexibility, assessment methods, learning opportunities, etc. In addition, words such as "co-op" and "applications" echo students' experience with recurring work terms. While further efforts and analysis are required to extrapolate key themes from students' comments (and at a more granular level), this initial probe highlights the potential benefit of using qualitative research methods to support the quantitative data.

We also calculate the rank distribution of number of words written by each student in Fig. 2 and 3 as a proxy measure for response quality. Note that the 2013 survey comments are unavailable and are therefore not included in Fig. 2 and 3. Student responses are seen to follow a decaying distribution on the log-log charts, with a few students writing a great deal and many students writing little or nothing. It can also be seen that the online administration has not appreciably changed the shape of this distribution, and has in fact increased the total number of words written (meaning that each student who wrote something tended to write more when completing the exit survey electronically). It can also be seen that ME and MTE student behaviours in terms of amount of words written is similar. Altogether, this suggests that online-inperson engineering exit surveys can be conducted with high completion rates, similar quality of responses, and a higher number of words written when compared to PAP exit surveys.

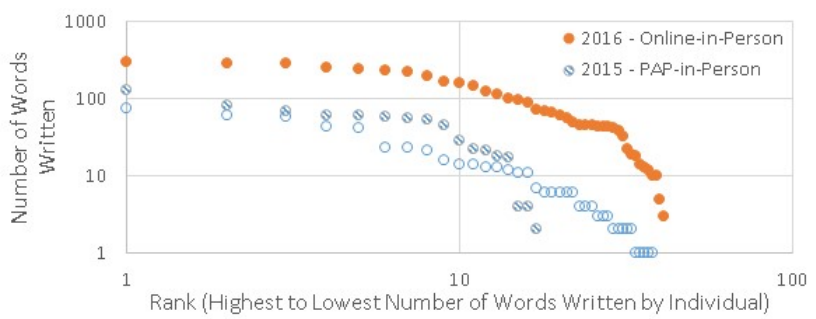

Fig. 2. Rank distribution of words written in general comments by ME 4B students.

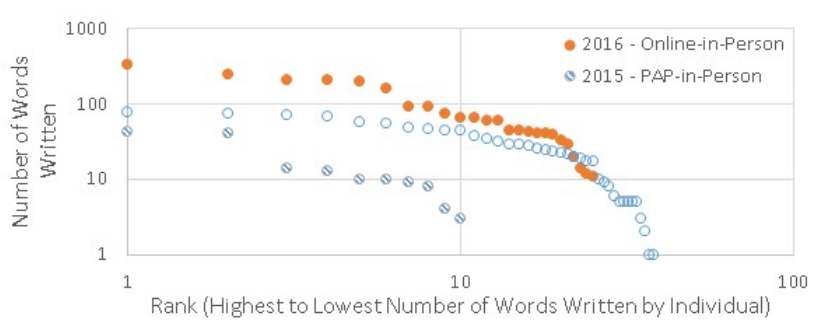

Fig. 3. Rank distribution of words written in general comments by MTE 4B students.

\subsection{Graduate Attribute Self-Assessment and Improvement}

We stress that the following analysis is preliminary and for piloting purposes. Given the response rates to the exit survey and the number of GA related questions each student received, we have not collected sufficient selfassessment responses from the $4 \mathrm{~B}$ students in each GA to draw firm conclusions. However, the goal of this work was to determine if the data collection method was sound, and if the GA self-assessment data collected will be useful moving forward.

We therefore explore two means of analyzing the collected 1A self-assessment, 4B self-assessment, and 4B rating of improvement data: comparison of $1 \mathrm{~A}$ and $4 \mathrm{~B}$ data, and consideration of the matched pair of $4 \mathrm{~B}$ selfassessment and perceived improvement. For the $1 \mathrm{~A} / 4 \mathrm{~B}$ comparison, a direct comparison of self-assessment responses can be made for a given GA. Keeping in mind that the cohorts are not the same (i.e we do not have matched pairs, and the respondents have had/will have different experiences of the program), and keeping in mind that the response to each indicator is an amalgam of many students responses to randomly assigned questions, Fig 4 shows one illustrative graph of such a comparison for the design attribute as self-assessed by MTE students.

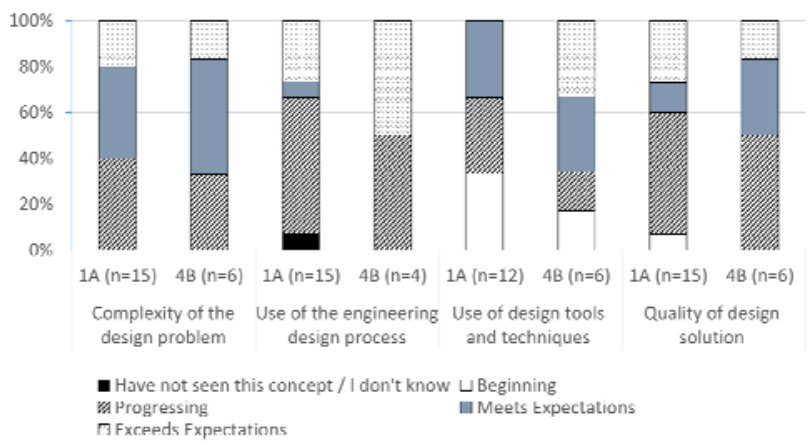

Fig. 4. Comparison of $1 A$ and $4 B$ MTE student selfassessment of the design graduate attribute.

It can be seen in Fig. 4 that students rate their capabilities in terms of the design process and use of tool and techniques as generally higher in $4 \mathrm{~B}$ than in $1 \mathrm{~A}$. Interestingly, it can be seen that a higher percentage of $4 \mathrm{~B}$ students rate themselves as exceeding expectations in use of the design process and use of design tools when compared to $1 \mathrm{~A}$ students. It is also interesting that a smaller number of 4B students rate themselves as exceeding expectations on complexity and quality of the design problem and solution (though more students rate themselves as meeting/exceeding in these indicators in $4 \mathrm{~B}$ compared to 1A). It should be noted that the small number of $4 \mathrm{~B}$ respondents means there are sizable uncertainties on the exact percentages of self-assessment. Regardless, the analysis shows that the tool can produce different measures of student self-assessment across the years, and suggests that the design complexity and quality indicators might bear further future study to inform the MME department as to the capabilities of our students.

We also present in Fig 5 a comparison of $1 \mathrm{~A}$ and $4 \mathrm{~B}$ MTE students in the communication GA. With the same 
cautions, it can be seen in Fig 5 that while $100 \%$ of six 4B students rate themselves as meeting or exceeding expectations in oral and graphical communication, 4B students rank themselves as little improved in terms of written communication and the ability to generate content. And a higher percentage of 4B students rate themselves as 'beginning' when it comes to clarity in communication. Other general areas where 4B ME and MTE students rate themselves more poorly include aspect of ethics (especially in handling ethical dilemmas), knowledge of tort and contract law, and impact of engineering on the society and environment (data not shown).

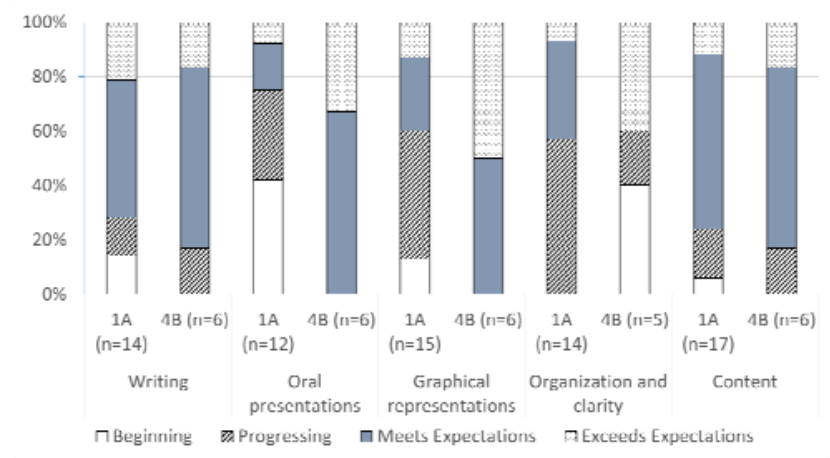

Fig. 5. Comparison of $1 A$ and $4 B$ MTE student selfassessment of the communication graduate attribute.

The 4B commination GA self-assessments suggest that $4 \mathrm{~B}$ students may well be more aware of the limits of their knowledge and abilities as compared to 1A students. This is indeed a desired and expected result of any educational process. To investigate this we present in Fig. 6 a comparison of the MTE 4B self-assessment of proficiency in the written communication and organization and clarity indicators of the communication GA along with the matched pair data of perceived improvement. In the bubble chart of Fig 6 the y-axis shows students self-assessment in the given indicator, while the $\mathrm{x}$-axis shows the perceived improvement since entering the program. The size of the bubble indicates the relative number of respondents.

Four broad regions of the graph in Fig. 6 can be defined. In the top right, students assess that they have improved over the course of the program to a high level of proficiency. This is obviously the desired goal of an educational program, and indeed 3 of the 6 students answering this question for written communication feel they have improved moderately or more and have achieved a reasonable (meeting expectations) level of proficiency. This infers that the $1 \mathrm{~A}$ students ranking themselves as similarly proficient to the $4 \mathrm{~B}$ students are likely unaware of their actual level of written communication proficiency, which we plan to investigate with direct measures in future studies. Meanwhile, 2 of 5 students feel even more positively about their improvement in organization and clarity (ranking themselves as improving a great deal to a level that corresponds to exceeding expectations.

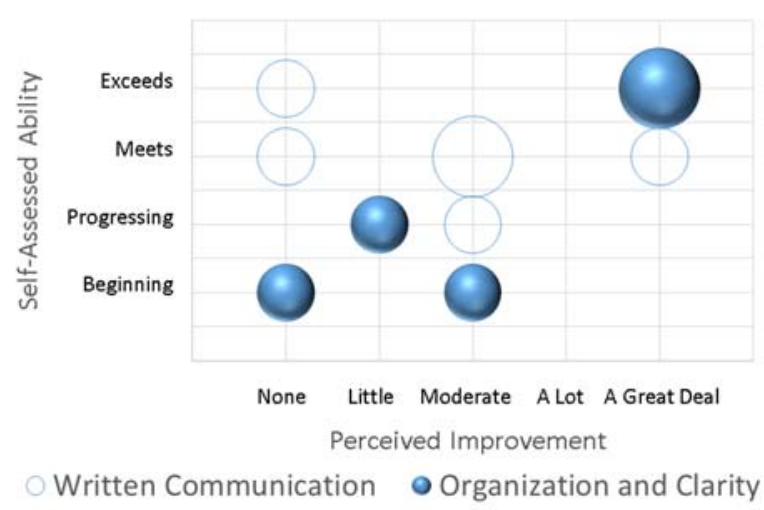

Fig. 6. Self-assessed ability versus perceived improvement over time spent in program for the written communication and organization and clarity indicators of the communication graduate attribute for $4 \mathrm{~B}$ MTE students

In the top left is a region where students feel they arrived in the program already proficient, and did not improve greatly while in the program. In Fig 2, we see that two students responded this way for written communication. Conversely, depending on students understanding of the question "Have you improved upon this skill during your time in the MTE program?" the students could be expressing that they improved despite/outside the MTE program.

The bottom left region of the graph illustrates GA/indicators for which the students feel they are not proficient and did not improve. No students responded this way for the written communication indicator, but students did respond this way for organization and clarity, which suggests that the students have at least learned what it is that they do not know. If results remain in this region over multiple more rigorous studies it suggests an area that the program could consider for improvement.

The final region (bottom right, indicating high improvement to a low level of proficiency) is one that the authours do not have an explanation for. It could perhaps indicate that students feel they have learned a great deal but feel that they still have a huge amount to learn. It could also indicate outliers of the data. Regardless, we have not seen any responses in this region in our analysis thus far.

\subsection{Rubric/Indicator Validation}

To evaluate the survey tool as well as the rubrics developed by the MME Department, all student groups were asked to rate the level of validity and clarity of GArelated questions based upon a 5-point Likert scale (from 'poor' to 'excellent' with 'I don't know' as a possible additional option). That is, students were asked to provide feedback on whether or not the questions reflected reasonable attributes of an engineer (validity) and the degree to which the questions made sense (clarity), and were primed with these definitions orally before each 
administration of the tool. The responses from all student groups are illustrated in Fig 7 and 8. In terms of validity, a similar percentage (slightly less than $10 \%$ ) of $1 \mathrm{~A}$ and $4 \mathrm{~B}$ ME students indicated 'I don't know' or 'poor,' with approximately $75-80 \%$ of both groups rating the validity as 'good' or higher (though again 4B students rated the validity of the measures as lower overall, with more responses in the 'good' rather than 'very good' or 'excellent' categories). It is worthy to note that the recent exposure to GAs given to the 1As by the Introduction to Engineering instructor may have had some bearing on the higher validity score. In contrast, the 4B MTE students provided a higher rating for validity than the 1A MTEs. This finding suggests that MTE students may have developed a more thorough understanding of graduate attributes of an engineer throughout their experiences in the program.

The overall scores for clarity were generally lower than the validity counterpart. This data is confounding as it poses the question of how students are able to thoughtfully rate the validity based upon disputably unclear questions. Approximately 50\% of the 4B ME students selected 'fair' or lower for clarity, whereas a slightly lower percentage (30\%) was given for the same response set from the $1 \mathrm{~A}$ MEs. In comparison, the 1A and 4B MTE groups provided a considerably high score for clarity, with more than $50 \%$ from both groups indicating 'good' or higher. While these results are shaped by students' varied exposure to GAs in the program, the authours acknowledge opportunities for improvement and the need to conduct ongoing analysis to identify which specific indicators were rated as unclear.

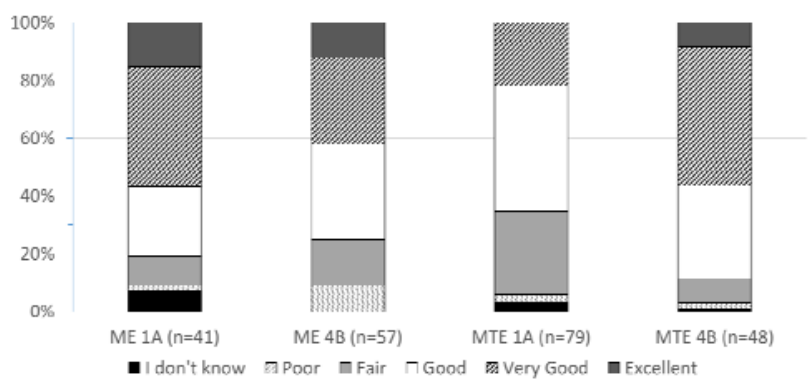

Fig. 7. Rating of GA question validity by $1 A$ and $4 B M E$ and MTE students.

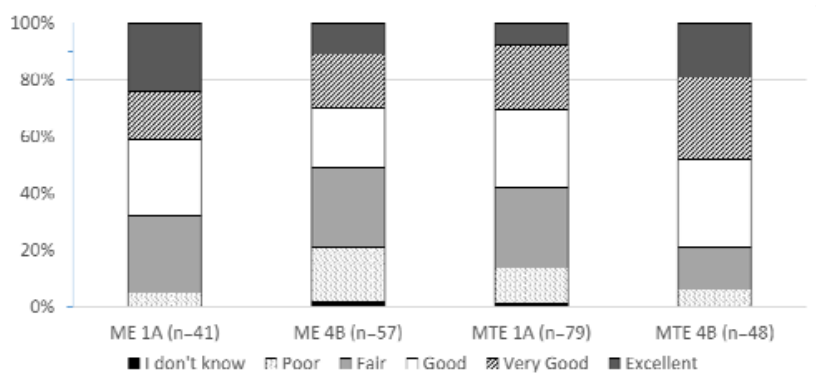

Fig. 8. Rating of GA question clarity by $1 A$ and $4 B M E$ and MTE students.

\subsection{Lessons Learned}

The ME and MTE 4B exit survey events were held during back-to-back pizza lunches followed by focus groups. While this format did not necessarily impact student attendance or response rates negatively, the authours suggest holding the events on two separate days to help alleviate stressors in the planning process. Another positive outcome of having designated dates for ME and MTE students is the presence of all organizers in facilitating and documenting feedback from focus groups One method of strategically restructuring the data collection process is to administer the online-in-person survey at the beginning of term during the mandatory safety lecture to increase response rates, with focus groups to follow at the end of term to provide feedback, connection to students, and interpretation of results. This would perhaps give the best of both worlds in that it would improve survey response rates while still gaining feedback on the 4B term (and concurrent end of the 8-month fourthyear-design-project).

Further, the authours intend, moving forward, to focus in on specific GAs for analysis on future surveys to increase granularity and statistical representation of responses. Based upon the data collected in the F'15 and W'16 pilot surveys, possible GAs to measure in-depth include knowledge base, ethics, communication, and impact of engineering. In this vein, concrete measures should be taken to yield higher response rates in coming terms. As evidenced by the $1 \mathrm{~A}$ and $4 \mathrm{~B}$ survey statistics presented in Table 1, very few students are likely to complete the survey outside a scheduled event. Thus, additional reminders and support from faculty members and professors would likely have a positive effect in increasing response rates in the future.

With regard to improving the survey tool itself, a possible starting point would be to refine the existing rubrics and indicators for clarity. This process entails reviewing the questions to ensure specific and measurable language is used and verifying clear distinctions between levels of achievement. Collaboration with on-campus resources (e.g. Student Success Office and other engineering departments) would be helpful in working towards more effective survey design, practices, and reward mechanisms. We also note that it may be wiser to ask 4B students to 'think back to $1 \mathrm{~A}$ and rate your younger self on the same scale', rather than asking for a level of improvement, since this should allow a more direct measure of the matched pairs of data (and might allow in future for a matched triplet of data of specific 1A student self-assessments, their matched assessments in 4B, and the retrospective consideration of their $1 \mathrm{~A}$ selves as 4B's). 


\section{Conclusions}

An (online survey-based) electronic self-assessment tool has been designed using rubric indicators and level descriptions to serve as an indirect measure of CEAB graduate attributes (GA). This tool was administered to $1 \mathrm{~A}$ Mechanical (ME) and Mechatronics (MTE) engineering students in the fall 2015 term, and to 4B ME and MTE students in the winter 2016 term. In the winter 2016 term the self-assessment tool was bundled into 4B exit survey, which was also administered electronically for the first time. The administration of both tools was generally online-in-person (that is, using an online platform, but administered in a physical classroom at a specified time), and paired with a pizza-lunch focus group session after the administration of the tool. This was done in order to increase participation rate and preserve as much as possible the environment of previous paper-and-pencil surveys administered in the Department.

In terms of responses rates and contents, it was found that the in-person aspect of the administration increased participation rates, but that the combination of survey, focus group, and pizza lunch in a separate time and place could not rival the participation rates for a survey presented without focus group and pizza during a mandatory lecture period. That said, the quality and quantity of responses to the survey were seen to not be damaged by the online administration, with similar themes emerging from this and previous PAP surveys, and with more words being written electronically by each student who chose to write comments, and a similar distribution of amount of words written by each student.

In terms of student self-assessment of GA, the preliminary/pilot results show that the tool can indeed bear fruit in giving varied responses across and within various GA, and between $1 \mathrm{~A}$ and 4B students (including suggestions that $4 \mathrm{~B}$ students indeed have learned while in the program and have come to recognize that their selfknowledge in 1A was incorrect). While low numbers of responses to the randomized GA questions limit statistical rigour, preliminary results suggest that GA's such as ethics, impact of engineering, communication, and knowledge base bear further study and consideration before taking potential actions to attempt to improve the program.

It is hoped that the results of the GA self-assessment and exit survey will lead to improvements of the ME and MTE programs at the University of Waterloo. The selfassessment tool will continue to undergo improvements and modifications and we plan to continue its use as part of the GA assessment and continual improvement process within the MME Department. It is hoped that this paper, and the tool it describes, will be of use to other programs considering similar endeavours, and the authors welcome communication and collaboration from interested parties.

\section{Acknowledgements}

We thank the Dean of Engineering's Office for purchasing the trial license of Qualtrics ${ }^{\mathrm{TM}}$ which allowed for the survey to be created and administered. We thank the Chair of the MME department and Head of the MTE program for their support in conducting and disseminating this work, for contributing funds to conduct the pizza lunch focus groups after each event, and for supporting attendance at CEEA16. We thank the professors of the 1A Introduction to Engineering courses for allowing us to contact their students during their class time. The administration of the tool and exit survey could not have occurred without the help of Tanya Yoworski and Carla Daniels (ME and MTE), and the support of the First-Year Engineering office and WEEF lab, who all have our thanks.

\section{References}

[1] Davidov, E., \& Depner, F. (2011). Testing for measurement equivalence of human values across online and paper-and-pencil surveys. Qual Quant, 45, 375-390.

[2] Easa, S. M. (2013). Framework and guidelines for graduate attribute assessment in engineering education. Canadian Journal of Civil Engineering, 40, 547-556.

[3] Huang, H. M. (2006). Do print and web surveys provide the same results? Computers in Human Behavior, 22, 334-350.

[4] Kongsved, S. M., Basnov, M., Holm-Christensen, K., \& Hjollund, N. H. (2007). Response rate and completeness of questionnaires: A randomized study of internet versus paperand-pencil versions. Journal of Medical Internet Research, 9.

[5] Musallam, A. B., Schallert, D. L., \& Kim, H. (2011). Do millennial undergraduates' views of writing differ when surveyed online versus on paper? Computers in Human Behavior, 27, 1915-1921.

[6] Nair, C. S., \& Adams, P. (2009). Forum survey platform: A factor influencing online survey delivery and response rate. Quality in Higher Education, 15, 291-296.

[7] Nulty, D. D. (2008). The adequacy of response rates to online and paper surveys: What can be done? Assessment \& Evaluation in Higher Education, 33, 301-314.

[8] Weigold, A., Weigold, I. K., \& Russell, E. J. (2013). Examination of the equivalence of self-reported survey-based paper-and-pencil and internet data collection methods. Psychological Methods, 18, 53-70.

[9] Wood, E., Nosko, A., Desmarais, S., Ross, C., \& Irvine, C. (2006). Online and traditional paper-and-pencil survey administration: Examining experimenter presence, sensitive material and long surveys. The Canadian Journal of Human Sexuality, 15, 147-155. 